\title{
Brecha digital, uso frecuente y aprovechamiento de Internet en México
}

\author{
Digital Divide, Frequently Use of Internet and \\ Benefits in Mexico
}

\author{
Djamel Toudert (D https://orcid.org/0000-0003-2833-4128 \\ El Colegio de la Frontera Norte, México,toudert@colef.mx
}

\begin{abstract}
Despite the need to validate the assumptions that intend to participate in the construction of the theory, the digital divide of frequent Internet users in the socioterritorial approach constitutes an unusual path. Considering this situation, this contribution has the objective of determining the validity of a multistage accessibility model moderated by the population size of Mexican localities. The research is based on microdata from the National Survey on Availability and Use of Information Technologies in Households, and for the validation of its hypothesis, it recurs to modeling with structural equations. Within the framework of this exploration, the multidimensionality and the high level of abstraction of the second-order constructs used in the research model are verified, revealing significant differences between groups of population size. Overall, the findings seem to show a frequent use that does not reflect in all cases skills of use and benefits use of the Internet.
\end{abstract}

Key words: digital divide, multistage accessibility, frequent users, beneficial use, locality size.

Resumen: El abordaje de la brecha digital en el contexto de los usuarios frecuentes de Internet constituye un acercamiento poco usual, a pesar de la necesidad de validar los supuestos socioterritoriales que pretenden participar en la construcción de su teoría. Con base en ello, este artículo tiene el objetivo de averiguar la validez de un modelo de accesibilidad moderado por el tamaño de las localidades donde habitan los usuarios mexicanos. La investigación se sustenta en microdatos de la Encuesta Nacional sobre Disponibilidad y Uso de las Tecnologías de la Información en los Hogares (Inegi, 2016a) y recurre a la modelación con ecuaciones estructurales para la validación de las hipótesis planteadas. Los resultados de la investigación dejan

Recepción:

$02 / 05 / 2018$

Aprobación:

$24 / 09 / 2018$ entrever una multidimensionalidad en el modelo socioterritorial, la cual es estructurada alrededor de diferencias significativas entre grupos locales y un uso frecuente que no parece asociado en todos los casos con habilidades y un aprovechamiento de Internet.

Palabras clave: brecha digital, accesibilidad multietápica, usuarios frecuentes, aprovechamiento del uso, tamaño de localidades. 


\section{Introducción}

Por lo general, la brecha digital ha sido un concepto genérico centrado en la disponibilidad y el acceso material de la población a Internet, posicionándose en la lógica de quienes la tienen y quienes no (Chen, 2003; Schleife, 2010; Warf, 2001). No obstante, más allá de la accesibilidad física a la red, otros acercamientos mostraron también una brecha digital dependiente de las habilidades y el aprovechamiento de los propios usuarios de Internet (DiMaggio et al., 2004; van Deursen et al., 2015b).

La apropiación final de Internet como un medio para obtener beneficios y ventajas, se convierte en un contexto de investigación para averiguar, entre otros, los límites y barreras que frenan este tipo de aprovechamiento. En estos ámbitos, los estudios han sido pocos, llegando a ser circunstanciales, y en cuanto a la exploración del impacto del tamaño de las localidades en la apropiación final de la red son prácticamente inexistentes (Blank y Groselj, 2014; Hargittai y Hinnant, 2008; van Deursen, 2015b). Más allá del reto intelectual de darle seguimiento al impacto de la ubicación en las etapas finales de la brecha digital, este conocimiento permite también diseñar políticas y estrategias localizadas, para la mejora del uso y el aprovechamiento de la red.

La especialización productiva de áreas específicas que favorece el desarrollo de las TIC, además de evidenciar espacios relativamente desconectados y olvidados, expresa también una relación de orden y de jerarquías en el caso de los territorios incorporados a la red (Castells, 2000; Gillespie y Williams, 1988). En el marco de estas relaciones e implicaciones, la validación de la investigación de los vínculos causales que inciden en la brecha digital puede recurrir a uno de los modelos teóricos, los cuales permiten explicar la apropiación final de Internet (Toudert, 2016; Tirado-Morueta et al., 2017; van Deursen y van Dijk, 2015).

En este sentido, el modelo accesibilidad multietápica constituye uno de los instrumentos más explicativos de las lógicas de uso y aprovechamiento de Internet, tanto por usuarios como de no usuarios. Esto le otorga a la apropiación de Internet una dimensión multietápica, que permite también el seguimiento de usuarios frecuentes de Internet, los cuales suelen estar excluidos generalmente de la población vinculada con la brecha digital.

El presente estudio tiene el objetivo de verificar, en primer lugar, la validez del modelo de accesibilidad multietápica, que posibilita explicar la apropiación final de Internet en el contexto de usuarios frecuentes en México. En seguida se analizará la consistencia moderadora de cuatro diferentes tamaños de localidades de residencia de usuarios implicados en estos procesos de apropiación de Internet. 


\section{Revisión de literatura e hipótesis}

\section{Usos de Internet: el tamaño poblacional de las aglomeraciones}

La importancia que puede tomar el tamaño de las localidades en la adopción y uso de Internet no fue considerada, por lo general, por la perspectiva prominente, como un factor de incidencia directa (Toudert, 2015; Shleife, 2010; Sinai y Waldfogel, 2004). Para dicha perspectiva, un posible impacto traduciría más bien, según Sinai y Waldfogel (2004) y Shleife (2010), el peso territorial de segmentos poblacionales específicos, que suelen influir en estas prácticas. Segmentos definidos por la proporción de la población de jóvenes, de estudiantes, de minorías étnicas, extranjeros, desempleados y empleados altamente calificados, entre otros, fueron puestos en evidencia por su incidencia en el tipo y las etapas de apropiación de Internet en diferentes escalas territoriales (Chen, 2013; Pick et al., 2015).

La investigación del impacto de la ubicación permitió vincular la adopción y el uso de Internet con diversas estructuras y tipos de organización territorial (Agarwal et al., 2005; Shleife, 2010; Chen, 2013; Pick et al., 2015; Pick et al., 2015; Shleife, 2010; Sinai y Waldfogel, 2004). En el marco de esta búsqueda, se exhibieron evidencias de la variación de la brecha digital en función de la ubicación vis a vis de las aglomeraciones urbanas y metropolitanas dominantes (Toudert, 2015; Grubesic, 2006; Chen, 2013; Pick et al., 2015). No obstante, los estudios que se enfocarían en la brecha digital desde la perspectiva del peso poblacional de las localidades han sido escasos y aún menos cuando se trata de la apropiación final de Internet por usuarios frecuentes (Toudert, 2015; Billón et al., 2008).

La incidencia del tamaño poblacional en el uso y adopción de Internet encuentra sentido, generalmente, en el crecimiento de los dividendos conforme a la ampliación del tamaño de la red, caracterizando de esta manera a lo que se conoce como efectos de red (Shleife, 2010; Goolsbee y Klenow, 2002). Dichos efectos fueron puestos en evidencia por: Goolsbee y Klenow (2002) en el caso de la difusión de las TIC por medio de los artefactos de acceso a Internet; en la toma de decisión de ingresar a la red por Agarwal et al. (2005); y en la probabilidad de volverse un usuario por Billón et al. (2008) y Shleife (2010).

De esta manera, el nivel de adopción de las TIC en una localidad parece jugar un papel trascendente en la difusión del uso hacia los demás habitantes no usuarios (Agarwal et al., 2005; Billón et al., 2008). Esto parece además superar la incidencia del tamaño del mercado en la disponibilidad de la 
infraestructura que condiciona la oferta del servicio de Internet, principalmente en países en desarrollo (Toudert, 2015; Pick et al., 2014).

Desde otra perspectiva, hay otras lecturas que se desprenden de las lógicas centro-periferia en materia de adopción y el uso de Internet, como un medio sustituto para compensar el alejamiento de los centros y/o complementario para potenciar las capacidades existentes (Billón et al., 2008; Shleife, 2010; Sinai y Waldfogel, 2004).

La función sustituta incide en localidades de baja densidad a través de la adopción de Internet, para remediar carencias de interacción y de accesibilidad a la oferta de la modalidad virtual, y la complementariedad intervendría en localidades de alta densidad para expandir y diversificar la oferta en la modalidad presencial. Desde esta lógica, las localidades de baja densidad son más propensas a la adopción de Internet cuando la evidencia parece apuntar más bien hacia una coexistencia de la sustitución y la complementariedad (Shleife, 2010; Sinai y Waldfogel, 2004).

\section{Respaldo teórico-conceptual del modelo de investigación}

El presente estudio será abordado por medio de una adaptación a las condiciones y necesidades de la investigación del modelo multifacético de acceso a Internet (Multifaceted Model of Internet Access), propuesto por van Deursen y van Dijk (2015). El modelo inicial de estos autores se sustentó en un concepto de brecha digital estructurada por las cuatro etapas sucesivas siguientes: acceso motivacional a Internet, acceso material a Internet, habilidades de acceso a Internet, y el acceso al uso provechoso de Internet. Dado que este artículo se centra exclusivamente en los usuarios frecuentes de Internet, se considera razonablemente cumplida la etapa motivacional por este tipo de usuarios, y por lo tanto, se descartará de este modelo de investigación.

\section{Acceso material a Internet}

Esta etapa de la brecha digital se enfoca a evidenciar las diferencias de disponibilidad de Internet, de los artefactos tecnológicos que permiten usarlo y los niveles de accesibilidad del usuario (Toudert, 2016; van Dijk, 2005; DiMaggio et al., 2004). Es el paso inicial hacia una apropiación de Internet plasmada en primer lugar por la posibilidad de conectarse a la red en los lugares de mayor estancia (casa, trabajo o escuela). La disponibilidad de la conexión en el conjunto de estos lugares fue traducida por la escasa literatura 
que se dedicó a estos temas, por una mayor disposición para el uso y aprovechamiento de Internet (Toudert, 2016; Zhao et al., 2010).

Del mismo modo, el acceso a diferentes artefactos tecnológicos (computadoras, tabletas, teléfonos y televisores inteligentes, entre otros) fue calificada de transcendental para la consulta de distintos tipos de contenido (van Deursen y van Dijk, 2015). En relación con estos aspectos, a pesar de los grandes avances en la difusión de Internet, la brecha digital sigue siendo importante, sobre todo entre quienes disponen de los artefactos y de la conexión a la red, y los que no la tienen (Toudert, 2015; Pick et al., 2014).

Estas diferencias se acentúan con el costo y la calidad de los servicios de Internet y de telefonía móvil, que son proporcionados de manera desigual, dependiendo de los planes contratados e inclusive dentro del mismo plan. A nivel de los usuarios, el conjunto de dichas diferenciaciones se expresa con una variación en la efectividad del servicio disponible y, desde luego, con su utilidad para el uso y aprovechamiento de Internet. Las restricciones que impone la variación de la calidad de la señal recibida tiende a fomentar la insatisfacción y el paso por malas experiencias, que suelen desanimar al usuario para profundizar en el uso de la red (Narteh, 2015; Meuter et al., 2000).

\section{Habilidades de acceso a Internet}

La estructura y el grado de sofisticación de los contenidos disponibles en Internet contribuyen a la segmentación de usuarios, en función de sus habilidades para acceder a ellos y ocuparlos para sacarles el mayor provecho (Hargittai, 2002; van Dijk, 2005). Estas habilidades del usuario definen lo que Hargittai (2002) y van Dijk (2005) caracterizaron como el segundo nivel de una brecha digital estructurada por varias determinantes de uso y aprovechamiento de Internet. En su conjunto, las habilidades determinantes se desprenden de la teoría cognitiva social y definen en gran medida una percepción de la capacidad individual para emprender acciones, que posibilitan lograr un objetivo autoasignado (van Deursen y van Dijk, 2009).

En la literatura de las desigualdades en el uso de Internet, las habilidades son autorreportadas por los mismos usuarios o medidas con pruebas directas (Hargittai y Hinnant, 2008; Hargittai, 2002; van Deursen y van Dijk, 2009). Estos dos abordajes concurren a relacionar actividades en línea con destrezas necesarias para llevarlas a cabo, como en el caso de actividades básicas para manejar el navegador, búsquedas y llenado de cuestionarios, las cuales requieren de habilidades operacionales. Pero también la 
navegación y la orientación necesitan de habilidades formales, la ubicación y la evaluación de la información por medio de habilidades informacionales, y finalmente, tomar el camino adecuado para alcanzar el objetivo asignado que exige un despliegue de habilidades estratégicas (Toudert, 2016; Tirado-Morueta et al., 2017; van Deursen y van Dijk, 2009).

Acceso al uso provechoso de Internet

Esta última etapa culmina la estructura conceptual de la brecha digital plasmando en diferentes finalidades pragmáticas las prácticas individuales de los usuarios. En este sentido, una vez que los usuarios traspasan las etapas anteriores de la brecha digital, tanto el tiempo y la frecuencia de uso como el tipo de actividades online se vuelven determinantes para acceder a beneficios y ventajas (Blank y Groselj, 2014; Hargittai y Hinnant, 2008; van Deursen et al., 2015b). A pesar de la poca investigación que se dedicó a estos aspectos, Zhao et al. (2010) mostraron, en el contexto educativo, que el tiempo y la frecuencia de conexión se vuelven incidentes en el aprovechamiento, cuando se toma en cuenta el lugar de la consulta (casa, escuela, cibercafés). Analizando el uso de Internet de la población alemana, van Deursen y van Dijk (2014) se percataron de que pasaban más tiempo libre en las redes sociales y actividades de ocio quienes tienen un nivel de educación bajo. En el mismo orden de ideas, el impacto de un nivel educativo creciente de los papás fue encontrado concluyente en el aprovechamiento escolar, inducido por las actividades online de estudiantes chinos (Zhao et al., 2010).

Estos hallazgos reflejan, según van Deursen y van Dijk (2015), la persistencia de las diferencias sociales que caracterizaron en el pasado a otros tipos de uso de la tecnología; además, Zhao et al. (2010) pusieron de relieve una prevalencia de la disparidad urbano-rural en el aprovechamiento escolar por medio de las actividades online. En el marco de estas consideraciones, el tiempo y la frecuencia de uso son esenciales para adquirir un conocimiento tácito y súper tácito que, una vez combinados con las adecuadas actividades online, se convierten en un vínculo de mayor aprovechamiento de los recursos de la red (Malecki, 2016).

Las mismas actividades son de por sí indicadores pertinentes de aprovechamiento, y por esta razón son conceptualmente sistematizadas y ordenadas en función de su incidencia potencial en el desempeño profesional, escolar o en otros ámbitos de la vida de los usuarios (Tirado-Morueta, 2017; van Deursen y van Dijk, 2015; Zhao et al., 2010). 
Hipótesis del modelo de investigación

La presente investigación retoma tres hipótesis principales del modelo multifacético de acceso a Internet, propuesto por van Deursen y van Dijk (2015), para analizar el efecto moderador del tamaño de las localidades en estas relaciones causales. El conjunto de dichas hipótesis se sostiene en las etapas sucesivas en la apropiación de Internet de van Dijk (2005), basada en la conceptualización de las brechas de acceso y de sus relaciones unas con otras (Ghobadi y Ghobadi, 2015). En el marco de este andamiaje conceptual, contar con el acceso material a Internet a través de diferentes plataformas y lugares de acceso fue hallado incidente en el desarrollo de las habilidades de los usuarios (Toudert, 2016; Tirado-Morueta, 2017; van Deursen y van Dijk, 2015; Zhao et al., 2010). Del mismo modo, la disponibilidad del acceso material a Internet fue encontrada con un impacto positivo y significativo en el acceso provechoso a Internet (Toudert, 2016; van Deursen y van Dijk, 2009 y 2015), que, a su vez, se benefició significativamente del impacto creciente de las habilidades de los usuarios (Toudert, 2016; van Deursen y van Dijk, 2015). Con base en estas exploraciones previas, se proponen las hipótesis siguientes:

$\mathrm{H} 1$ : El incremento en el acceso material a Internet impacta de manera positiva y significativa las habilidades de acceso de Internet.

H2: La ampliación del acceso material a Internet incide de manera positiva y significativa en el acceso al uso provechoso de Internet.

H3: La disponibilidad creciente de habilidades de acceso a Internet genera un incremento positivo y significativo en el acceso al uso provechoso de Internet.

Al tomar en cuenta que no existe aun suficiente evidencia directa para validar la mediación del tamaño poblacional en las relaciones causales del modelo de investigación, el estudio se propuso abordar este aspecto. Basándose en la verificación cruzada de trabajos anteriores de Agarwal et al. (2005), Billón et al. (2008), Shleife (2010) y Goolsbee y Klenow (2002), la mediación del tamaño poblacional tendría más posibilidad de ser concluyente, caracterizándose por diferencias significativas de las relaciones $\mathrm{H} 1$, $\mathrm{H} 2$ y $\mathrm{H} 3$. 


\section{Metodología y datos}

\section{Instrumento y muestreo}

Esta investigación ocupó microdatos de la "Encuesta Nacional sobre Disponibilidad y Uso de las Tecnologías de la Información en los Hogares (ENDUTIH-2015)", que aplicó el Instituto Nacional de Estadística y Geografía (Inegi) del 8 de junio al 31 de julio de 2015 (Inegi, 2016a). La encuesta fue aplicada en 90,030 viviendas y se entrevistaron a 292,055 personas de más de seis años de edad, distribuidas en los 32 estados de la República, para lograr una estimación de proporciones de $1 \%$, un nivel de confianza del $90 \%$, un error relativo máximo de $10.48 \%$ y una tasa de no respuesta de 15\% (Inegi, 2016a).

El 61.80\% de la población entrevistada resultó usuaria de Internet, de la cual 114,334 personas lo ocupan a diario (Inegi, 2016b). Es precisamente estos usuarios frecuentes de Internet quienes constituyen la población objetivo del estudio, y sus respuestas al cuestionario aplicado conforman los datos involucrados en la evaluación del modelo de investigación que exhibe la Figura 1.

\section{Caracterización de variables y escalas de medición}

La diversidad de la estructura de datos de la encuesta analizada requirió usar las técnicas PLS (Partial Least Squares), que permiten incorporar a diferentes dimensiones y escalas de medición (Falk y Miller, 1992). Lo anterior, además de evitar adecuaciones pesadas que exigiría otro método, como el basado en el análisis de covarianzas, posibilita operar en el contexto de latentes formativas, con datos que carecen de una distribución normal y un contexto teórico en etapas de construcción (Chin, 1998).

El modelo de investigación propuesto incluye 15 variables manifiestas, cuatro de ellas relacionadas con el constructo: habilidades de acceso a Internet, como indicadores reflectivos (véase Tabla 2). Las variables manifiestas restantes son incorporadas a dos constructos de segundo orden: acceso material a Internet y acceso al uso provechoso de Internet. En su conjunto, el modelo de investigación fue examinado en el contexto de cuatro grupos de localidades definidas por el número de sus habitantes: G1 (más de 100,000), G2 (de 15,000 a 99,999), G3 (de 2,500 a 14,999), G4 (menos de 2,500).

1 La figura y las tablas se encuentran en el Anexo, al final del presente artículo (Nota del editor). 
Las mediciones para caracterizar a las dimensiones del estudio fueron posibles gracias a la adaptación a los datos de la encuesta. Varias de estas dimensiones analizadas fueron también involucradas en otros estudios (Toudert, 2016; Lee, 2009; Min, 2010; Suárez et al., 2016; van Deursen et al., 2014; van Deursen y van Dijk, 2015). El acceso material a Internet fue caracterizado como un constructo bidimensional de segundo orden, compuesto por el acceso material y la satisfacción del acceso. El acceso material fue expresado por dos ítems: el equipo utilizado y el lugar de acceso a Internet. El ítem equipo utilizado fue medido como en van Deursen y van Dijk (2015). La satisfacción con el acceso fue medido con la evaluación de la calidad de los servicios de telefonía e Internet, aplicando cuatro puntos de la escala Likert (desde excelente hasta pobre). El constructo habilidades de acceso a Internet fue caracterizado en el presente estudio por los ítems habilidades operacionales, habilidades formales, habilidades informacionales y habilidades estratégicas, que fueron utilizados por van Deursen et al., (2014) y van Deursen et al., (2012). El acceso al uso provechoso de Internet se abordó como un constructo de segundo orden de tres dimensiones, que fueron definidas en van Deursen y van Dijk (2015), Tirado-Morueta et al. (2017) y van Deursen et al. (2015b).

\section{Resultado: validez y solidez del modelo}

Los usuarios diarios de Internet que se describen en la Tabla 1, se encuentran de manera dominante en localidades de más de 100,000 habitantes (74\%), obedecen a una distribución de género similar a la observada en la población nacional y se concentran en edades oscilantes entre 6 y 35 años (64\%). Son distribuidos de una manera homogénea entre los niveles de estudio básico, preparatoria y universidad, y son mayoritariamente ocupados como empleados o estudiantes (véase Tabla 1). En términos de dinámica de usuarios de Internet, se confirma el hecho de que si las diferencias de género han ido achicándose en México, para las demás variables estructurales de la brecha digital las desigualdades siguen siendo a veces muy pertinentes (Toudert, 2015 y 2016).

La evaluación del modelo de investigación se realizó tomando en cuenta las recomendaciones de Henseler et al. (2016), que permitieron lograr condiciones de robustez del proceso de validación PLS, que consta en la evaluación sucesiva del modelo general, del modelo de medidas y del modelo estructural. 
El modelo general presenta una discrepancia geodésica $d_{G}$ y una discrepancia de mínimos cuadrados no ponderados de la bondad del ajuste $d_{U L S}$ debajo de las discrepancias del modelo actual en un nivel del 95\% (Dijkstra y Henseler, 2015a). La importancia de estas discrepancias fue evaluada por medio del criterio aproximado de ajuste del modelo medido con el residuo cuadrado de la raíz estandarizada (SRMR), revelando un valor de 0.04, el cual se encuentra debajo del valor del límite aceptado de 0.08 , que nos indica un ajuste pertinente del modelo de investigación (Hu y Bentler, 1999).

La evaluación del modelo de medidas exhibe valores de carga de ítems de constructos reflectivos que se presentan en la Tabla 2, con cifras más altas que la regla de admisión de 0.7 (Nunnally y Bernstein, 1994). Esto en conjunto con la consistencia de la fiabilidad interna que muestra a valores del indicador rho ( $p$ A) de Dijkstra-Henseler superiores a la cifra recomendada de 0.7, confirmando la robustez de los constructos reflectivos (Dijkstra y Henseler, 2015b). Respecto a los constructos formativos, tanto los pesos como los signos son los adecuados, significativos y definidos por un factor de inflación de la varianza (VIF), que permite descartar los inconvenientes de multicolinealidad (Henseler et al., 2016).

La validez convergente del modelo de investigación fue evaluada con la varianza promedio extraída (AVE), mostrando valores superiores a 0.5 que identifican esos factores, exhibiendo a más de la mitad de la varianza de sus indicadores (Fornell y Larcker, 1981). La validez discriminante se evaluó con el ratio de correlaciones heterotrait-monotrait (HTMT), que muestra en la Tabla 3 valores inferiores a uno e indiquen a una pertinente discriminación entre factores (Hensler $e t$ al., 2015).

La evaluación del modelo estructural destaca valores de la $\mathrm{R}^{2}$ ampliamente superiores a $10 \%$ de la varianza, explicada por la fluctuación de las variables exógenas, lo cual se considera una tasa aceptable en estados preliminares de investigación (Falk y Miller, 1992). De la misma manera, las variables latentes endógenas reflejan un poder de predicción satisfactorio del modelo, que alcanza a expresar en el caso del valor más alto de $\mathrm{R}^{2}$ a la casi totalidad de la varianza explicada por el constructo: acceso al uso provechoso de Internet (véase Tabla 2).

El nivel de significación de las hipótesis del modelo de investigación fue estimado con el método bootstrap de 5000 re-muestreo; las tres relaciones evaluadas $\mathrm{H} 1, \mathrm{H} 2, \mathrm{H} 3$ y sus efectos totales resultaron todos concluyentes con $\mathrm{P}<0.001$ (véase Tabla 4). Igualmente, la relación entre los constructos de segundo orden: acceso material a Internet y acceso al uso provechoso de Internet, y sus efectos totales resultaron todos significativos 
$(\mathrm{P}<0.001)$. El mismo nivel de significación fue también observado en el caso del efecto indirecto calculado para los tres constructos de primer orden con el resto de las variables latentes del modelo de investigación con $\mathrm{P}<0.01$ y $\mathrm{P}<0.001$.

La evaluación del efecto de moderación que genera el tamaño de las localidades de residencia se realizó por medio de un análisis multigrupo, aplicando la prueba de diferencia de grupos de Henseler (2007). En los resultados que se presentan en la Tabla 5 , la relación entre el acceso material a Internet y las habilidades de acceso a Internet exhibe diferencias significativas $(\mathrm{P}<0.10)$, para el comparativo entre grupos G1-G4, G2-G4 y G3-G4, siendo G2-G4 el comparativo que muestra las diferencias más fuertes.

Para la relación entre el acceso material a Internet y el acceso al uso provechoso de Internet las diferencias son significativas $(\mathrm{P}<0.10)$ únicamente para G1-G2; mientras tanto, para la relación entre las habilidades de acceso y el acceso provechoso a Internet son los comparativos G2-G3 y G2-G4 los que exhiben las diferencias significativas $(P<0.10)$ con un efecto más fuerte en G2-G3.

\section{Discusión}

Tomando en cuenta que se usaron por primera vez componentes jerárquicos en un modelo multifacético de acceso a Internet, los resultados de la investigación ratifican la multidimensionalidad del alto nivel de abstracción de los conceptos de segundo orden utilizados. Para el constructo de segundo orden: acceso material a Internet, las dimensiones acceso material y satisfacción con el acceso mostraron una incidencia significativa y positiva, con un mayor impacto de la primera dimensión mencionada (0.7117).

En este sentido, es importante recordar que la dimensión de acceso material fue por sí sola la latente que caracterizó el mismo concepto en Tirado-Morueta et al. (2017) y van Deursen y van Dijk (2015), quienes la hallaron concluyente en un modelo similar. No obstante, la agregación, en el presente caso, de una nueva dimensión centrada en la satisfacción del usuario con los servicios de voz y datos brindó una mayor definición y una incidencia igual de alta $(0.5757)$.

Lo mismo parece desprenderse del constructo de segundo orden: acceso al uso provechoso de Internet; sus tres dimensiones fueron también encontradas con un impacto significativo y una incidencia de involucramiento en transacciones y actividades comerciales más alta, comparado con las que generan las demás dimensiones. De hecho, la dimensión involucrada en 
transacciones y actividades comerciales (0.7174) mostró un impacto en el uso provechoso de Internet de casi tres veces más importante que las dimensiones: usos productivos $(0.293)$ y experiencia de uso $(0.2412)$.

Esto parece indicar que en el contexto de los usuarios frecuentes mexicanos, lo que más pesa en el aprovechamiento de Internet es el involucramiento en compras y pago en línea. Este comportamiento fue asociado con usuarios generalmente de estatus alto, quienes son más propensos al desarrollo de actividades comerciales online (Hargittai y Hinnant, 2008; Madden y Lee, 2003). Sin embargo, van Deursen et al. (2015b) revelaron que en el contexto de la población alemana estas actividades obedecen a características evolutivas, donde dominan en los inicios hombres jóvenes, un nivel educativo bajo y un ingreso suficiente.

Tomados en conjunto, estos hallazgos parecen indicar un involucramiento en actividades comerciales y transacciones incidentes en el uso provechoso de Internet, que puede considerarse como contextual y temporal. Pero este no es el caso de las dimensiones de uso productivo y experiencia en el uso, que fueron encontradas en otros trabajos con un desempeño similar al presente estudio (van Deursen y van Dijk, 2015; van Deursen et al., 2015b).

Desde otra perspectiva, a sabiendas de que el modelo multifacético de acceso a Internet fue ideado para el seguimiento de la brecha digital en la población en general (van Deursen y van Dijk, 2015), se corrobora en este artículo su validez nomológica y estructural para el estudio de usuarios frecuentes de Internet. De hecho, todas las relaciones causales del modelo de investigación $(\mathrm{H} 1, \mathrm{H} 2$ y H3) fueron halladas altamente significativas, confirmando que el uso frecuente de Internet no permite saltar en todos los casos las barreras sucesivas de la accesibilidad, de habilidades y de uso provechoso de la red. Desde la perspectiva aclaratoria, este tipo de apropiación de Internet admite diversas explicaciones, las cuales se discutirán enseguida las más pertinentes.

Estudios anteriores reportaron que personas con un nivel educativo bajo usan Internet por periodos largos durante su tiempo libre; usuarios que se caracterizan, en su mayoría, por un nivel socioeconómico bajo, quienes suelen utilizar la red de manera superficial (van Deursen y van Dijk, 2013; van Dijk, 2005). En comparación, DiMaggio et al. (2004) mostraron que los usuarios de estatus socioeconómico alto usan Internet de forma más productiva, confirmando en cierta medida lo observado por Hargittai y Hinnant (2008), quienes hallaron que los más educados suelen ocupar Internet en actividades que permiten incrementar su capital. 
En este sentido, se considera que estos factores que limitan el aprovechamiento de Internet en otros contextos son susceptibles de generar una incidencia similar en el paisaje de usos en México. A título indicativo, cabe mencionar que durante 2015, cerca de la mitad de los usuarios frecuentes de Internet en México contaron con una escolaridad que llegaba, en su nivel más alto, hasta la secundaria (véase Tabla 1 ).

Además de los factores señalados antes, otros aspectos que presentan similitudes con evidencias halladas en las brechas de conocimiento fueron identificados por su incidencia en la brechas del uso de la red (Hargittai y Hinnant, 2008; van Deursen y van Dijk, 2015). El género y la edad son factores que favorecen las brechas del uso, gracias al tipo de actividades online elegidas por unos y otros.

A título de ejemplo, el uso provechoso, como la búsqueda de información sobre salud y comercio interesaría principalmente a hombres de edad madura, en comparación con un uso centrado en el entretenimiento y comunicación, el cual se relaciona por lo general con mujeres y jóvenes (Madden y Lee, 2003; van Deursen y van Dijk, 2013). Desde esta perspectiva, en el caso de asistir a un achicamiento de la brecha digital hombre/mujer en México, es importante subrayar que un poco más del $60 \%$ de los usuarios frecuentes tienen menos de 35 años, lo cual estaría a favor del desarrollo de actividades menos productivas online (véase Tabla 1 ).

Otro aspecto que parece incidir en la variación de los usos es la limitación de las diferentes plataformas tecnológicas utilizadas para ingresar a la red. Comparado con la utilización de computadoras, Pearse y Rice (2013) observaron que los móviles inteligentes reducen el acceso a actividades online benéficas para los usuarios armenios. Se esperaría también un impacto similar en México, ya que el $87 \%$ de los usuarios frecuentes de Internet ocupan su teléfono inteligente como medio principal para el acceso a la red (Inegi, 2016a).

Respecto a la incidencia de residir en aglomeraciones de distintos tamaños poblacionales, se identificaron diferencias significativas entre cada uno de los tres primeros grupos de localidades y el último grupo de menos de 2,500 habitantes, en lo correspondiente a la relación entre el acceso material y las habilidades de acceso a Internet. Estas últimas aglomeraciones de menor peso poblacional son, por lo general, localidades típicas de áreas rurales, las cuales se caracterizan en el contexto mexicano por su dispersión y a veces por su aislamiento (Toudert, 2015).

En estos espacios periféricos, tanto el acceso a Internet como las condiciones de desarrollo de las habilidades necesarias son diferentes, en 
comparación con regiones de una influencia urbana consecuente (Malecki, 2003; Shleife, 2010). De hecho las diferencias en la provisión del servicio de Internet, la estructura demográfica de las localidades, las condiciones de educación, la ocupación y la posición en el trabajo son, entre otros, aspectos determinantes en la segmentación del uso de la red entre áreas urbanas y rurales (Agarwal et al., 2005; Chen, 2013; Pick et al., 2015).

Tomando en cuenta estos elementos de explicación, es importante insistir en que habitar en una área rural no significa en todos los contextos, como lo menciona Shleife (2010) para el caso alemán, una diferencia significativa en el uso de Internet comparado con áreas urbanas. No obstante, en el caso chino, Zhao et al. (2010) encontraron dicha diferencia como significativa y proporcional al grado de desarrollo de las localidades. Estos hallazgos que, según parece están ligados a los niveles de desarrollo, exigen profundizar en la investigación comparativa, para aclarar, entre otros, la incidencia del acceso a Internet en la manifestación de habilidades, en especial en los distintos contextos de integración territorial de áreas rurales.

Las diferencias, entre grupos de localidades, del impacto del acceso material a Internet en las habilidades de acceso y el uso provechoso de la red, involucran para el primer impacto a los grupos de localidades G1-G4, G2G4 y G3-G4. Estos hallazgos son sinónimos de un impacto diferenciado y no lineal del tamaño poblacional en las relaciones causales del modelo de investigación. Del total de las relaciones causales analizadas, solo algunas presentan un impacto concluyente localizado entre los grupos G1, G2, y G3 con G4 específicamente. En el marco de esta relación causal (H1), el grupo de localidades con la población más baja se caracteriza por diferencias significativas con todos los demás grupos con una población superior.

Estos resultados parecen indicar, para los grupos G1, G2 y G3, un impacto comparable que no se sostiene para el grupo G4 por las carencias de artefactos y servicios, que permiten tanto la accesibilidad a Internet como el desarrollo de habilidades para su uso. Desde esta perspectiva, el grupo G4 parece conformar en México un umbral poblacional para la accesibilidad a Internet y el desarrollo de habilidades de uso. Respecto a la incidencia del acceso material a Internet en el uso provechoso de la red $(\mathrm{H} 2)$, la reducción del tamaño poblacional de las localidades en el grupo G2 genera diferencias significativas con el grupo G1, y estas parecen endosadas a un cambio pertinente en las condiciones de aprovechamiento del uso en G2.

Esto puede ser indicativo de marginación en educación y empleo, propio de las lógicas de estructuración de las localidades del grupo G2, pues estas condiciones dejan de incidir en los demás grupos caracterizados por 
localidades de menos habitantes. El tamaño poblacional parece ceder el paso ante la proporción de habitantes que cuenta con los servicios y artefactos de acceso a Internet, pero no los ocupan de manera provechosa. Dicho de otro modo, las localidades del grupo G2 parecen concentrar, más que en ningún otro grupo, las deficiencias relacionadas con las condiciones de uso que no son sujetas sensu estricto a la evolución lineal de la población.

La misma lógica parece afectar las diferencias en el impacto de las habilidades de acceso a Internet en el uso provechoso de la red (H3), entre el grupo G2 y G3 y el grupo G2 y G4. Para esta relación causal, las deferencias parecen obedecer a un efecto escalonado del tamaño poblacional, que se encuentra limitado en su nivel más bajo por el grupo G2. Esto quiere decir que si el grupo G4 constituye un umbral poblacional para la accesibilidad en México, el grupo G2 es otro umbral para el desarrollo de habilidades que posibilitan un uso provechoso de Internet.

\section{Conclusión}

La complejidad del abordaje de la brecha digital y sus diferentes aproximaciones imponen una exploración epistemológica continua de los supuestos que pretenden participar en la construcción de su teoría. En el marco de este esfuerzo, el presente estudio validó satisfactoriamente el uso de componentes de orden superior en la modelación multietápica de la brecha digital. Los resultados confirman por primera vez la multidimensionalidad y el alto nivel de abstracción de los constructos de segundo orden: acceso material a Internet y acceso al uso provechoso de la red. Dichos constructos fueron fortalecidos ocupando nuevas dimensiones como la satisfacción del usuario y la experiencia de uso, que permitieron lograr un oportuno planteamiento nomológico del modelo de investigación. En este caso de estudio, la disponibilidad del acceso material a Internet y la satisfacción con el servicio resultaron de alto impacto en la accesibilidad; mientras, el uso comercial fue altamente incidente en el aprovechamiento del uso de la red.

Tomando en cuenta que el modelo multifacético de acceso a Internet fue propuesto para el análisis de la accesibilidad y el aprovechamiento de Internet de la población en general, su aplicación en el contexto de usuarios frecuentes en México mostró también una validez significativa de todas las hipótesis planteadas. De esta manera, el uso frecuente de Internet no parece traducirse en todos los casos en habilidades de uso y aprovechamiento de la red. Estos aspectos parecen variar en función de las características socioeconómicas de los usuarios y las plataformas tecnológicas elegidas para acceder a la red. 
Desde la perspectiva del tamaño de las localidades donde habitan los usuarios frecuentes, se encontraron diferencias significativas de la incidencia del acceso material a Internet en las habilidades de acceso a la red, entre los tres grupos con mayor población y el último que abarca a localidades rurales o periféricas de menos de 2,500 habitantes. Estas diferencias parecen reflejar más bien a las variaciones estructurales que caracterizan las localidades urbanas y metropolitanas vis a vis de los espacios periféricos.

De la misma manera, se revelaron también otras diferencias entre grupos de tamaño de localidades, que parecen fundamentadas principalmente por los niveles de integración y el posicionamiento orgánico inherentes a los sistemas urbanos y metropolitanos. En este sentido, las diferencias detectadas entre el acceso material a Internet y el acceso al uso provechoso de la red, por un lado, y entre las habilidades de acceso y el acceso al uso provechoso, por el otro, son ilustrativas del impacto sistémico que atraviesa la trama territorial más allá de la variación del tamaño poblacional de sus localidades.

Es importante subrayar la aparición, en los hallazgos de la presente investigación, de un umbral para la accesibilidad en las localidades con menos de 2,500 habitantes, y otro para la incidencia de las habilidades en el uso provechoso de Internet en las localidades de 15,000 a 99,000 habitantes.

En conjunto, estos resultados parecen indicar la necesidad de continuar la investigación de la apropiación final de Internet, poniendo, a futuro, una atención especial en los diferentes contextos de integración territorial.

\section{Referencias}

Agarwal, Ritu et al. (2005), "Social interactions and the 'digital divide': explaining regional variations in Internet use", en Information Systems Research, vol. 20, núm. 2, Estados Unidos: Informs.

Billon, Margarita et al. (2008), “The Spatial Distribution of the Internet in the European Union: Does Geographical Proximity Matter?”, en European Planning Studies, vol. 16, núm. 1, Reino Unido: Taylor \& Francis. DOI: 10.1080/09654310701748009.

Blank, Grant y Groselj, Darja (2014), "Dimensions of Internet use: amount, variety, and types”, en Information, Communication y Society, vol. 17, núm. 4, Reino Unido: Taylor \& Francis. DOI: 10.1080/1369118X.2014.889189.

Castells, Manual (2000), The rise of the network society, Reino Unido: Blackwell.

Chen, Wenhong (2013), "The implications of social capital for the digital divides in America”, en The Information Society, vol. 29, núm. 1, Reino Unido: Taylor \& Francis. DOI: $10.1080 / 01972243.2012 .739265$.

Chin, Wynne (1998), “The partial least squares approach to structural equation modeling”, en George, Marcoulides [ed.], Modern Methods for Business Research, Estados Unidos: Lawrence Erlbaum Associates Publisher. 
Dijkstra, Theo y Henseler, Jörg (2015a), "Consistent and asymptotically normal PLS estimators for linear structural equations”, en Computational Statistics y Data Analysis, vol. 81, Reino Unido: Elsevier. DOI: 10.1016/j.csda.2014.07.008.

Dijkstra, Theo y Henseler, Jörg (2015b), "Consistent partial least squares path modeling”, en MIS Quarterly, vol. 39, núm. 2, Estados Unidos: Carlson School of Management.

DiMaggio, Paul et al. (2004), "From unequal access to differentiated use: A literature review and agenda for research on digital inequality”, en Neckerman, Kathryn [ed.], Social inequality, Estados Unidos: Russell Sage Foundation.

Falk, Franc y Miller, Nancy (1992), A primer for soft modeling, Estados Unidos: The University of Akron Press.

Fornell, Claes y Larcker, David (1981), "Evaluating structural equation models with unobservable variables and measurement error", en Journal of Marketing Research, vol. 1, núm. 1, Estados Unidos: American Marketing Association.

Gillespie, Alex y Williams, Helen (1988), “Telecommunications and the reconstruction of regional comparative advantage”, en Environment and Planning A, vol. 20, núm. 10, Estados Unidos: Sage.

Ghobadi, Sharhla y Ghobadi, Zahra (2015), "How access gaps interact and shape digital divide: A cognitive investigation”, en Behaviour \& Information Technology, vol. 34, núm. 4, Estados Unidos: Taylor \& Francis.

Goolsbee, Austan y Klenow, Peter (2002), "Evidence on learning and network externalities in the diffusion of home computers", en The Journal of Law and Economics, vol. 45, Estados Unidos: National Bureau of Economic Research.

Grubesic, Tony (2006), "A spatial taxonomy of broadband regions in the United States", en Information Economics and Policy, vol. 18, Reino Unido: Elsevier. DOI: 10.1016/j. infoecopol.2006.05.001.

Hargittai, Eszter y Hinnant, Amanda (2008), "Digital inequality: Differences in young adults' use of the Internet”, en Communication Research, vol. 35, núm. 5, Estados Unidos: Sage.

Hargittai, Eszter (2002), "Second-level digital divide: Differences in people's online skills", en First Monday, vol. 7, núm. 4. Disponible en: http://www.firstmonday. org/ojs/ index.php/fm/article/view/942 [20 de marzo de 2015].

Henseler, Jörg et al. (2016), "Using PLS path modeling in new technology research: updated guidelines", en Industrial Management y Data Systems, vol. 116, núm. 1, Estados Unidos: Emerald Publishing Limited. DOI: 10.1108/IMDS-09-2015-0382.

Henseler, Jörg et al. (2015), "A new criterion for assessing discriminant validity in variancebased structural equation modeling", en Journal of the Academy of Marketing Science, vol. 43, núm. 1, Estados Unidos: Springer International Publishing.

Henseler, Jörg (2007), "A new and simple approach to multi-group analysis in partial least squares path modeling”, en Herald, Martens y Termos, Næs [eds.], Causalities explored by indirect observation: Proceedings of the 5th international symposium on PLS and related methods (PLS'07), Noruega: Universite of Twente.

$\mathrm{Hu}, \mathrm{Li}$-tze y Bentler, Peter (1999), "Cutoff criteria for fit indexes in covariance structure analysis: conventional criteria versus new alternatives", en Structural Equation Modeling: a multidisciplinary Journal, vol. 6, núm. 1, Reino Unido: Taylor \& Francis. DOI:10.1080/10705519909540118. 
Inegi (2016a), Encuesta Nacional sobre Disponibilidad y Uso de las Tecnologias de la Información en los Hogares (ENDUTIH-2015): Diseño muestral, México: Instituto Nacional de Estadística y Geografía.

Inegi (2016b), Encuesta Nacional sobre Disponibilidad y Uso de las Tecnologias de la Información en los Hogares (ENDUTIH-2015): Cuestionario, México: Instituto Nacional de Estadística y Geografía.

Lee, Seongil (2009), "Mobile Internet Services from Consumers' Perspectives", en International Journal of Human-Computer Interaction, vol. 25, núm. 5, Reino Unido: Taylor \& Francis. DOI: 10.1080/10447310902865008.

Madden, Mary y Lee, Rainie (2003), America’s Online Pursuits, Estados Unidos: Pew Internet and American Life Project.

Malecki, Eduward (2016), "Real people, virtual places, and the spaces in between", en Socio-Economic Planning Sciences, vol. 58, Reino Unido: Elsevier. DOI: 10.1016/j. seps.2016.10.008.

Malecki, Edward (2003), "Digital development in rural areas: potentials and pitfalls", en Journal of Rural Studies, vol. 19, núm. 2, Reino Unido: Elsevier. DOI: 10.1016/S07430167(02)00068-2.

Meuter, Mattheuw et al. (2000), "Self-Service Technologies: Understanding Customer Satisfaction with Technology-Based Service Encounters", en Journal of Marketing, vol. 64, núm. 3, Estados Unidos: American Marketing Association.

Min, Seong-jae (2010), "From the Digital Divide to the Democratic Divide: Internet Skills, Political Interest, and the Second-Level Digital Divide in Political Internet Use", en Journal of Information Technology y Politics, vol. 7, núm. 1, Reino Unido: Taylor \& Francis. DOI: 10.1080/19331680903109402.

Narteh, Bedman (2015), "Perceived service quality and satisfaction of self-service technology: The case of automated teller machines", en International Journal of Quality y Reliability Management, vol. 32, núm. 3, Reino Unido: Emerald Publishing Limited. DOI: 10.1108/IJQRM-08-2012-0113.

Nunnally, Jum y Bernstein, Ira (1994), Psychometric Theory, Estados Unidos: McGraw-Hill. Pearse, Katy y Rice, Ronald (2013), "Digital Divides From Access to Activities: Comparing Mobile and Personal Computer Internet Users", en Journal of communication, vol. 63, Reino Unido: Wiley Online Library. DOI: 10.1111/jcom.12045.

Pick, James et al. (2015), "United States digital divide: State level analysis of spatial clustering and multivariate determinants of ICT utilization", en Socio-Economic Planning Sciences, vol. 49, Reino Unido: Elsevier. DOI: 10.1016/j.seps.2014.09.001.

Pick, James et al. (2014), "Broadband utilization in the Indian states: socio-economic correlates and geographic aspects", en Jyoti, Choudrie y Middleton, Catherine [eds.], Management of broadband technology innovation, Reino Unido: Routledge.

Schleife, Katrin (2010), "What really matters: Regional versus individual determinants of the digital divide in Germany", en Research Policy, vol. 39, Reino Unido: Elsevier. DOI: 10.1016/j.respol.2009.11.003.

Sinai, Todd y Waldfogel, Joel (2004), "Geography and the Internet: is the Internet a substitute or a complement for cities?”, en Journal of Urban Economics, vol. 56, núm. 1, Reino Unido: Elsevier. DOI: 10.1016/j.jue.2004.04.001.

Suárez, David et al. (2016), "Satisfaction of business customers with mobile phone and Internet services in Spain”, en Telecommunications Policy, vol. 40, Reino Unido: Elsevier. DOI: 10.1016/j.telpol.2015.10.002. 
Tirado-Morueta, Ramón et al. (2017), "Empirical study of a sequence of access to Internet use in Ecuador", en Telematics and Informatics, vol. 34, Reino Unido: Elsevier. DOI: 10.1016/j.tele.2016.12.012.

Toudert, Djamel (2016), “Teoría del recurso y la apropiación: un acercamiento empírico a partir de las etapas del modelo de acceso digital en México", en Acta Universitaria, vol. 26, núm. 4, México: Universidad de Guanajuato. DOI: 10.15174/au.2016.875.

Toudert, Djamel (2015), "Brecha digital y marginación socioterritorial: el caso de Mexico", en Garrocho, Carlos y Buzai, Gustavo [eds.], Geografía Aplicada en Iberoamérica. Avances, retos y perspectivas, México: El Colegio Mexiquense.

Toudert, Djamel (2014), "Aprovechamiento de las TIC en México: una aproximación empírica a través del uso de microdatos y la aplicación de la modelación PLS”, en Apertura, vol. 6, núm. 1, México: Universidad de Guadalajara.

Toudert, Djamel (2013), "La brecha digital en los contextos de marginación socioterritorial de las localidades mexicanas de más de 2500 habitantes: Exploración y discusión”, en Comunicación y Sociedad, núm. 19, México: Universidad de Guadalajara.

van Deursen, Alexander y van Dijk, Jan (2015), “Toward a Multifaceted Model of Internet Access for Understanding Digital Divides: An Empirical Investigation”, en The Information Society, vol. 31, núm. 5, Reino Unido: Taylor \& Francis. DOI: 10.1080/01972243.2015.1069770.

van Deursen, Alexander et al. (2015b), "Increasing inequalities in what we do online: A longitudinal cross sectional analysis of Internet activities among the Dutch population (2010 to 2013) over gender, age, education, and income", en Telematics and Informatics, vol. 32, Reino Unido: Elsevier. DOI: 10.1016/j.tele.2014.09.003.

van Deursen, Alexander et al. (2014), "Internet Skills, Sources of Support, and Benefiting From Internet Use”, en International Journal of Human-Computer Interaction, vol. 30, núm. 4, Reino Unido: Taylor \& Francis. DOI: 10.1080/10447318.2013.858458.

van Deursen, Alexander y van Dijk, Jan (2013), “The digital divide shifts to differences in usage”, en New media y society, vol. 16, núm. 3, Estados Unidos: Sage.

van Deursen, Alexander et al. (2012), "Proposing a survey instrument for measuring operational, formal, information and strategic Internet skills", en International Journal of Human-Computer Interaction, vol. 28, Reino Unido: Taylor \& Francis. DOI: 10.1080/10447318.2012.670086.

van Deursen, Alexander y van Dijk, Jan (2009), "Using the Internet: Skill related problems in users' online behavior”, en Interacting with Computers, vol. 21, Reino Unido: Elsevier. DOI: $10.1016 /$ j.intcom.2009.06.005.

van Dijk, Jan (2005), The deepening divide: Inequality in the information society, Reino Unido: Sage.

Warf, Barney (2001), "Segueways into cyberspace: multiple geographies of the digital divide. 2001", en Environment and Planning B: Planning and Design, vol. 28, núm. 1, Estados Unidos: Sage.

Zhao, Ling et al. (2010), "Internet inequality: The relationship between high school students' Internet use in different locations and their Internet self-efficacy", en Computers $y$ Education, vol. 55, Reino Unido: Elsevier. DOI: 10.1016/j.compedu.2010.05.010. 
Anexo

Figura 1

Propuesta de modelo de investigación

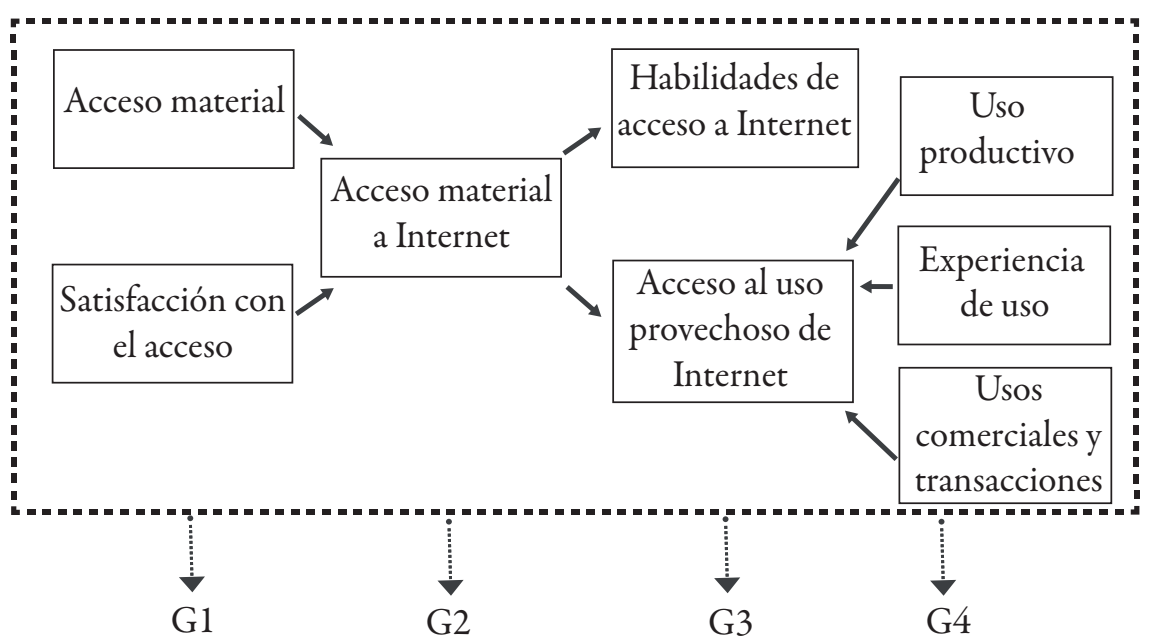

Fuente: Elaboración propia. 


\section{Tabla 1}

\section{Características sociodemográficas de la población objetivo}

\begin{tabular}{|c|c|c|c|}
\hline Género & $\%$ & Ocupación & $\%$ \\
\hline Hombres & 48.61 & Estudiante & 30.56 \\
\hline Mujeres & 51.39 & Empleado & 37.70 \\
\hline Rangos de edad (en años) & & Obrero & 0.60 \\
\hline 6 a 12 & 20.33 & Autoempleado & 9.70 \\
\hline 13 a 18 & 12.15 & Dueño de negocio & 1.41 \\
\hline 19 a 35 & 31.05 & Trabajador sin remuneración & 1.25 \\
\hline 36 a 45 & 15.47 & Otros & 18.78 \\
\hline 46 a 55 & 11.58 & Tamaño de localidades & \\
\hline 56 a 64 & 5.41 & 100,000 y más habitantes & 74.12 \\
\hline 65 y más & 4.01 & De 15,000 a 99,999 habitantes & 12.40 \\
\hline Nivel educativo & & De 2,500 a 14,999 habitantes & 8.47 \\
\hline Primaria & 23.13 & Menos de 2,500 habitantes & 5.00 \\
\hline Secundaria & 25.05 & Muestreo & \\
\hline Preparatoria & 22.84 & Tamaño del muestreo total & 292,055 \\
\hline Universidad & 24.91 & Tamaño del muestreo objetivo & 114,334 \\
\hline Posgrado & 2.45 & & \\
\hline Otros & 1.62 & & \\
\hline
\end{tabular}

Fuente: Elaboración propia. 


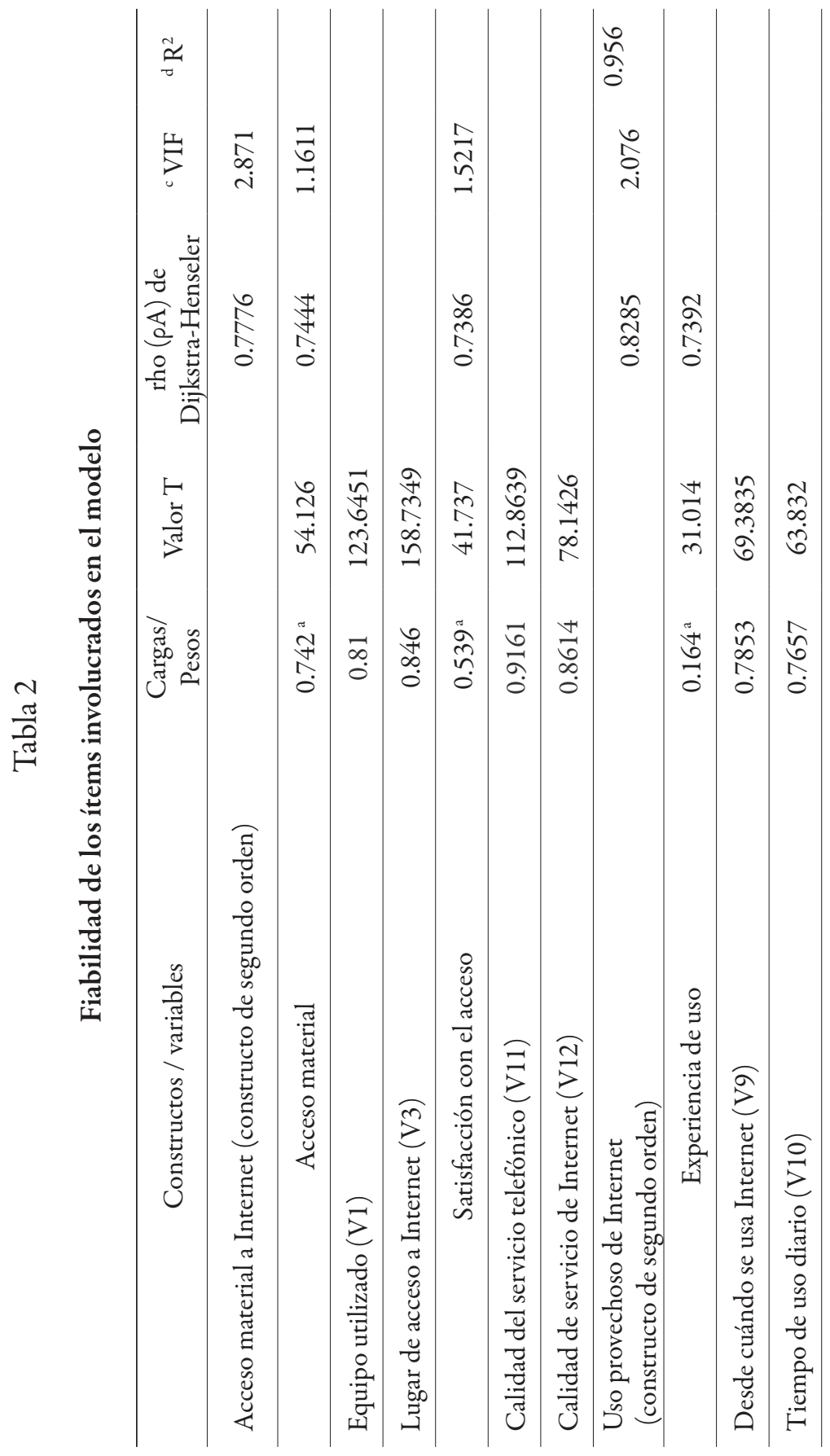




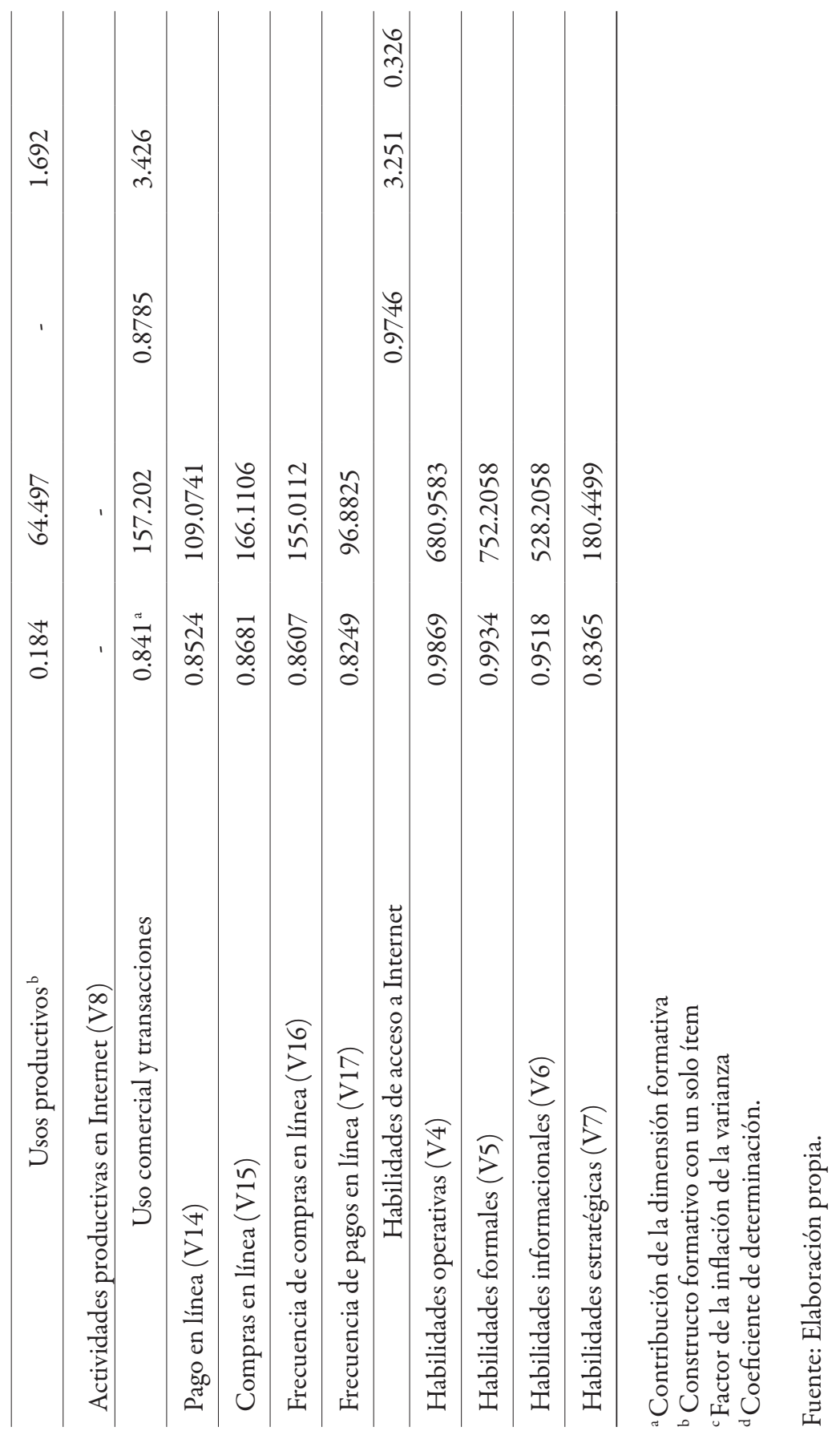




\section{Tabla 3}

La validez convergente y discriminante (AVE y HTMT)

\begin{tabular}{lllllllll}
\hline Constructos & AVE* & (a) & (b) & (c) & (d) & (e) & (f) & (g) \\
\hline (a) & 0.4375 & & & & & & & \\
\hline (b) & 0.6861 & 0.6449 & & & & & & \\
\hline (c) & 0.7928 & 0.7101 & 0.3118 & & & & & \\
\hline (d) & 0.9159 & 0.6117 & 0.7812 & 0.187 & & & & \\
\hline (e) & 0.4719 & 0.6282 & 0.7576 & 0.2277 & 0.801 & & & \\
\hline (f) & 0.6013 & 0.7387 & 0.621 & 0.2436 & 0.7078 & 0.6653 & & \\
\hline (g) & - & 0.5879 & 0.7605 & 0.1719 & 0.7911 & 0.7461 & 0.7063 & \\
\hline (h) & 0.732 & 0.432 & 0.4964 & 0.1762 & 0.5412 & 0.6823 & 0.4737 & 0.467 \\
\hline
\end{tabular}

*promedio de la varianza extraída.
(a) Acceso material a Internet
(b) Acceso material
(c) Satisfacción con el acceso
(d) Habilidades de acceso a Internet
(e) Acceso al uso provechoso de Internet
(f) Experiencia de uso
(g) Usos productivos
(h) Uso comercial y transacciones

Fuente: Elaboración propia. 


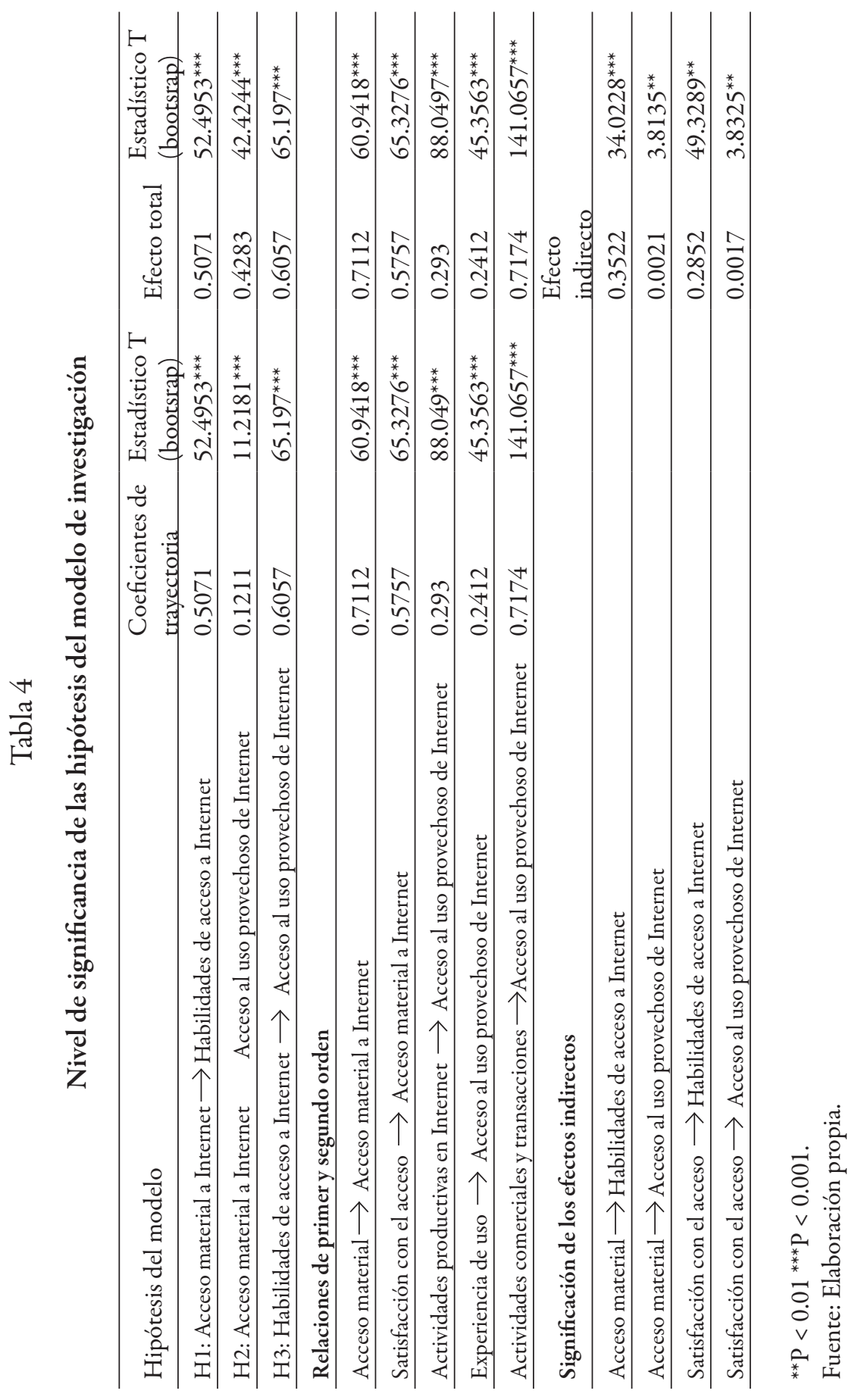




\section{Tabla 5}

\section{Resultados de la prueba de análisis multigrupo}

\begin{tabular}{ccc}
\hline Relaciones & Comparativo & P de Henseler \\
\hline Acceso material a Internet & Habilidades de acceso a Internet \\
\hline & G1-G2 & 0.289434 \\
\hline G1-G3 & 0.307346 \\
\hline G1-G4 & $0.908512^{*}$ \\
\hline G2-G3 & 0.486656 \\
\hline G2-G4 & $0.930562^{*}$ \\
\hline G3-G4 & $0.917208^{*}$ \\
\hline Acceso material a Internet & Acceso al uso provechoso de Internet \\
\hline G1-G2 & $0.952481^{*}$ \\
\hline G1-G3 & 0.743175 \\
\hline G1-G4 & 0.818947 \\
\hline G2-G3 & 0.218699 \\
\hline G2-G4 & 0.404219 \\
\hline G3-G4 & 0.646438 \\
\hline G1-G2 & 0.000317 \\
\hline G1-G3 & 0.158557 \\
\hline G1-G4 & 0.146726 \\
\hline G2-G3 & $0.936919^{*}$ \\
\hline G2-G4 & $0.912529^{*}$ \\
\hline G3-G4 & 0.461196 \\
\hline
\end{tabular}

*significación a 0.10 .

Fuente: Elaboración propia. 
Djamel Toudert. Doctor por el Instituto de Altos Estudios de América Latina (Universidad de París 3) y Profesor-investigador del departamento de Estudios Urbanos y del medio Ambiente de El Colegio de la Frontera Norte, México. Principales líneas de investigación: la apropiación socioterritorial de las tecnologías de la información y las comunicaciones, y gestión y promoción de destinos. Publicaciones recientes: Toudert, Djamel (2018), "Connectivité et isolement dans le monde scolaire mexicain", en La revue Communication. technologies et développement, núm. 5. Disponible en: http://wp.comtecdev.com/wp-content/uploads/2018/01/Toudert.pdf; Toudert, Djamel y Bringas-Rábago, Nora (2018), "El impacto del apego al equipo en el consumo recreativo de los espectadores transfronterizos de fútbol", en Retos, núm. 33. Disponible en: https://recyt.fecyt.es/index.php/ retos/article/view/53659/35605; Toudert, Djamel y Bringas-Rábago, Nora (2017), "Reciprocity between soccer events and visited destination in a border context", en Journal of Travel \& Tourism Marketing, Reino Unido: Taylor \& Francis. DOI: 10.1080/10548408.2017.1401029. 